\title{
Enhanced mPGES-1 Contributes to PD-Related Peritoneal Fibrosis via Activation of the NLRP3 Inflammasome
}

\begin{abstract}
Qimei Luo ${ }^{1+}$, Qinghua Hu ${ }^{1 \dagger}$, Qingkun Zheng ${ }^{1}$, Lewei Gong ${ }^{1}$, Lijuan Su ${ }^{1}$, Baojun Ren ${ }^{2}$, Yongle $\mathrm{Ju}^{2}$, Zhanjun Jia ${ }^{3 * t}$ and Xianrui Dou ${ }^{1 * t}$
\end{abstract}

'Department of Nephrology, Shunde Hospital, Southern Medical University (The First People's Hospital of Shunde), Foshan, China, ${ }^{2}$ Department of Gastrointestinal Surgery, Shunde Hospital, Southern Medical University (The First People's Hospital of Shunde), Foshan, China, ${ }^{3}$ Nanjing Key Laboratory of Pediatrics, Children's Hospital of Nanjing Medical University, Nanjing, China

OPEN ACCESS

Edited by:

Rujun Gong,

University of Toledo Medical Center,

United States

Reviewed by:

Guangyu Zhou,

Shengjing Hospital of China Medical

University, China

Lihong Chen,

Dalian Medical University, China

${ }^{*}$ Correspondence:

Zhanjun Jia

jiazj72@hotmail.com

Xianrui Dou

betty9911@163.com

†These authors have contributed equally to this work

Specialty section:

This article was submitted to Nephrology

a section of the journal

Frontiers in Medicine

Received: 03 March 2021

Accepted: 26 March 2021

Published: 18 May 2021

Citation:

Luo Q, Hu Q, Zheng Q, Gong L, Su L,

Ren B, Ju Y, Jia Z and Dou X (2021)

Enhanced mPGES-1 Contributes to

$P D$-Related Peritoneal Fibrosis via

Activation of the NLRP3

Inflammasome.

Front. Med. 8:675363.

doi: 10.3389/fmed.2021.675363
Background: Microsomal prostaglandin E synthase-1 (mPGES-1)-derived prostaglandin $E_{2}$ (PGE2) is a chief mediator of inflammation. However, the role and mechanism of mPGES-1 in peritoneal dialysis (PD)-associated peritoneal fibrosis have not been investigated.

Material and Methods: In PD patients, mPGES-1 expression in peritoneum tissues and the levels of PGE2, IL-1 $\beta$, and IL-18 in the dialysate were examined. In rat peritoneal mesothelial cells (RPMCs), the regulation and function of mPGES-1 and NLRP3 inflammasome were investigated. The expression of extracellular matrix proteins and the components of NLRP3 inflammasome were detected by Western blotting or real-time quantitative PCR.

Results: In PD patients with ultrafiltration failure (UFF), mPGES-1 was enhanced in the peritoneum, which was associated with the degree of peritoneal fibrosis. Accordingly, the intraperitoneal PGE2 levels were also positively related to the PD duration, serum C-reactive protein levels, and serum creatinine levels in incident PD patients. In RPMCs, high-glucose treatment significantly induced mPGES-1 expression and PGE2 secretion without affecting the expressions of mPGES-2 and cPGES. Inhibition of mPGES-1 via short hairpin RNA significantly ameliorated the expression of extracellular matrix proteins of RPMCs induced by high glucose. Additionally, high glucose markedly activated NLRP3 inflammasome in RPMCs that was blunted by mPGES-1 inhibition. Furthermore, silencing NLRP3 with siRNA significantly abrogated the expression of extracellular matrix proteins in RPMCs treated with high glucose. Finally, we observed increased $\mathrm{IL}-1 \beta$ and IL-18 levels in the dialysate of incident PD patients, showing a positive correlation with PGE2.

Conclusion: These data demonstrate that mPGES-1-derived PGE2 plays a critical role in PD-associated peritoneal fibrosis through activation of the NLRP3 inflammasome. Targeting mPGES-1 may offer a novel strategy to treat peritoneal fibrosis during PD.

Keywords: mPGES-1, PGE2, peritoneal fibrosis, inflammation, NLRP3 inflammasome 


\section{INTRODUCTION}

Peritoneal dialysis (PD) has become a well-established treatment option for patients with end-stage renal disease due to the advantages of its minimal requirements for technical support and better preservation of residual renal function (1). However, longterm PD is limited because chronic exposure to bioincompatible PD fluid can lead to obvious peritoneal fibrosis (2). These changes in the peritoneum include the loss of the mesothelial monolayer, thickening of the submesothelial layer because of increased deposition of the extracellular matrix, and angiogenesis (3-5). Fibrosis of the peritoneal membrane can culminate in peritoneum ultrafiltration failure (UFF), eventually forcing the withdrawal of PD therapy (6).

Accumulating evidence has implicated that sterile PD solutions containing high glucose can induce chronic sterile inflammation in the submesothelial zone, leading to peritoneal fibrosis $(7,8)$. High levels of inflammatory cytokines in the peritoneal cavity seem to be associated with the progress of peritoneal fibrosis (9). The fibrotic progress develops following chronic sterile inflammation in the peritoneum. As the first protection against biochemical and microorganism attack, peritoneal mesothelial cells incur various injuries and secrete inflammatory cytokines or chemokines, leading to the synthesis of extracellular matrix proteins and progression to peritoneal fibrosis $(10,11)$. Sustained peritoneal chronic inflammation is considered a potential mechanism of peritoneal fibrosis (12). Targeting regulation of peritoneal chronic inflammation may be an effective anti-fibrosis therapy in PD.

Prostaglandin $\mathrm{E}_{2}$ (PGE2) is an abundant prostanoid that is considered the chief mediator of inflammation. The biosynthesis of PGE2 is catalyzed by prostaglandin E synthases, that contain microsomal prostaglandin E synthase-1 (mPGES1), microsomal prostaglandin $\mathrm{E}$ synthase-2 (mPGES-2) and cytosolic prostaglandin E synthase (cPGES). mPGES-2 and cPGES are constitutively expressed, whereas mPGES1 , which is the dominant synthetic enzyme for PGE2 production, is an inducible enzyme under many pathological conditions (13). mPGES-1 is involved in inflammation, pain, angiogenesis, and tumorigenesis (13-15). Evidence has shown that mPGES-1 is a critical mediator of chronic inflammation in animal models of arthritis (16). High glucose increases PGE2 synthesis in human peritoneal mesothelial cells (17), while the role of mPGES-1-derived PGE2 in highglucose-induced peritoneal mesothelial cell injury remains to be defined.

Recently, the NOD-like receptor family, pyrin domaincontaining 3 (NLRP3) inflammasome was found to be activated in the peritoneal tissues of acute bacterial peritonitis in patients on PD, and blockade of NLRP3 rescued morphologic alterations during acute peritonitis (18). The NLRP3 inflammasome plays a critical role in the acute inflammation response of the peritoneum, but the role of the NLRP3 inflammasome in the condition of chronic inflammation in the peritoneum still needs to be explored. More interestingly, PGE2 activates NLRP3 signaling in peritoneal macrophages (19). However, it remains unknown whether $\mathrm{mPGES}-1 / \mathrm{PGE} 2$ mediates activation of the NLRP3 inflammasome under the condition of chronic inflammation induced by PD fluid.

In the present study, we examined the expression of mPGES-1 in the peritoneum and the secretion of PGE2 in PD fluid and analyzed the correlation between the activation of mPGES-1/PGE2 and peritoneal fibrosis. Additionally, the effect of mPGES-1/PGE2 on the regulation of the NLRP3 inflammasome and cascade in high-glucose-induced fibrosis was analyzed.

\section{MATERIALS AND METHODS}

\section{Human Sample Collection}

Peritoneum tissues from inguinal hernia patients with normal renal function, ESRD patients, and PD patients with UFF were collected at Shunde Hospital of Southern Medical University. Enrollment included patients aged 14 years and older who were diagnosed with hernia repair and needed to accept surgery therapy or ESRD patients or patients who had withdrawn from PD therapy due to UFF. Patients were excluded if they refused to give written consent or had malignant disease or had diabetes mellitus or had heterotopia endometriosis or took non-steroidal anti-inflammatory drugs (NSAIDs) in the past 2 weeks or took COX-2-selective inhibitors (e.g., celecoxib, nimesulide, meloxicam, and rofecoxib) in the past 2 weeks. Ultrafiltration failure was defined as, after a 4 -h residence period with $4.25 \%$ PD fluid, the net ultrafiltration failure to achieve at least $400 \mathrm{~mL}$ (20). Peritoneal biopsies were collected at the time of laparoscopic inguinal hernia repair or laparoscopic for PD catheter implantation or catheter withdrawal. Peritoneal tissues of inguinal hernia patients with normal renal function were normal control samples. We also collected PD fluid samples and serum samples from 116 incident PD patients. The daily ultrafiltration volume and daily urine volume were recorded, and the ultrafiltration function of the peritoneal membrane was evaluated by carrying out 4 -h PET. The nocturnal peritoneal dialysate samples and serum samples were collected on the day of PET. The peritoneal dialysate was drained after at least $8 \mathrm{~h}$ of dwell exchange. Serum C-reactive protein, serum hemoglobin, serum creatinine, serum calcium, dialysate creatinine, and dialysate urea nitrogen levels were assessed using an automatic biochemical instrument (AU5800; Beckman Coulter). All the patients provided informed consent. The use of human biopsy samples, serum and dialysate samples was approved by the Ethical Review Board of Shunde Hospital of Southern Medical University.

\section{Histology and Immunohistochemistry}

Changes in the peritoneal tissues were assessed using paraformaldehyde-fixed, paraffin-embedded sections (4$\mu \mathrm{m}$ ) with hematoxylin-eosin staining (HE) or Masson's trichrome staining (Masson). The thickness of the peritoneum was determined under a microscope and was expressed as the mean of five independent measurements for each section. Immunohistochemistry was examined in paraffin sections using a microwave-based antigen retrieval technique (21). Briefly, sections $(4-\mu \mathrm{m})$ were deparaffinized, rehydrated and incubated 
in $3 \% \mathrm{H}_{2} \mathrm{O}_{2}$ for 15 min to block endogenous peroxidase. Antigen retrieval for mPGES-1 from the sections was performed using an antigen retrieval solution $(1 \mathrm{mmol} / \mathrm{L}$ Tris-HCL, $0.1 \mathrm{mmol} / \mathrm{L}$ EDTA, $\mathrm{pH}$ 8.0) for $15 \mathrm{~min}$ at high power in a microwave oven. Then, sections were incubated with 5\% normal goat serum (abs933, Absin, China) in PBS for 60 min. Subsequently, sections were incubated overnight at $4^{\circ} \mathrm{C}$ with a rabbit antimPGES-1 antibody (no. ab203247, Abcam, England) or a isotype IgG (no. 3900s, Cell Signaling Technology), followed by the incubation with secondary antibody at room temperature for $1 \mathrm{~h}$, and the signal was visualized using a DAB kit (no. G1215-1, Servicebio, China). The optical density of mPGES-1 in the peritoneum was quantified using Image-Pro plus software. Five consecutive fields were assessed for each section, and a mean value from five independent measurements was used for statistical analysis.

\section{ELISA}

The PGE2, IL-1 $\beta$, and IL-18 protein levels were quantified using commercial kits (no. ml024761-C, ml050857-C, ml058055C, respectively; Shanghai Enzyme-linked Biotechnology, China) following the manufacturer's instructions. PGE2 was measured in 48-h conditioned medium.

\section{Cell Culture}

The immortalized rat peritoneal mesothelial cell (RPMC) line was from Zongpei Jiang (The Sixth Affiliated Hospital, Sun Yat-sen University, Guangzhou, China). Cells were cultured in DMEM/F12 medium containing 10\% fetal bovine serum (FBS; Gibco, USA) and $1 \%$ antibiotics $(100 \mu \mathrm{g} / \mathrm{mL}$ of streptomycin and $100 \mathrm{U} / \mathrm{mL}$ of penicillin) (Life Technologies, USA) at $37^{\circ} \mathrm{C}$ with $5 \% \mathrm{CO}_{2}$. Cells were deprived of serum for $24 \mathrm{~h}$ and were stimulated with D-glucose (Life Technologies, USA) at a normal (5.5 mmol/L) or high (138 mmol/L) concentration for $0,24,48$, and $72 \mathrm{~h}$.

\section{Transient Transfection}

RPMCs were seeded in a six-well plate at $70 \%-80 \%$ confluence before transfection. The cells were transfected with mPGES1 shRNA (GeneCopoei, Guangzhou, China) or NLRP3 siRNA (RiboBio, Guangzhou, China) or a negative control of shRNA or siRNA using Lipofectamine 3000 (Life Technologies, USA) according to the manufacturer's instructions. In brief, for mPGES-1 shRNA plasmid transfection, the cells were transfected with 2 ug mPGES-1 shRNA plasmid or control plasmid $8 \mathrm{~h}$ before high glucose treatment. For NLRP3 siRNA transfection, the cells were incubated with $500 \mathrm{nM}$ NLRP3 siRNA or scrambled siRNA, then the media were replaced with D-glucose at a high concentration $6 \mathrm{~h}$ after transfection. Next, the cells were harvested for mRNA or protein analysis at $24 \mathrm{~h}$ or $48 \mathrm{~h}$ after high glucose stimulation.

\section{RNA Extraction and Quantitative Real-Time PCR}

Total RNA was extracted from the cultured cells with Trizol reagent (Invitrogen, USA). cDNA was synthesized from 1 $\mu \mathrm{g}$ of total RNA using the PrimeScript ${ }^{\mathrm{TM}} \mathrm{RT}$ reagent Kit
TABLE 1 | Primer sequences for q-PCR.

\begin{tabular}{lc}
\hline Gene symbol & Primer sequences \\
\hline$\beta$-actin & 5'-TGTGACGTTGACATCCGTAAAG-3' \\
& 5'-GGCAGTAATCTCCTTCTGCATC-3' \\
mPGES-1 & 5'-AAGCCTGGCCATCTGTATT-3' \\
& 5'-TGAGCCAGATTGTACCACTTC-3' \\
mPGES-2 & 5'-GAGAAAGCTCGCAACAACTAAAT-3' \\
cPGES & 5'-TCATGGCTGGGTAGTAGGT-3' \\
& 5'-CTTGTCAGTGTCCAGGTGTAT-3' \\
IL-18 & 5'-TTCTCGTCCTCCATGCTAAG-3' \\
& 5'-GAATCCCAGACCAGACTGATAAT-3' \\
NLRP3 & 5'-GGTAGACATCCTCCATCCTTC-3' \\
& 5'-GGAAGATGTGGACCTCAAGAAA-3' \\
Caspase-1 & 5'-GATCCAAGTGATCTGCCTTCTC-3' \\
& 5'-AAGACAAGCCCAAGGTATCA-3' \\
& 5'-AAGAATCCCTCTCGGAGTTC-3'
\end{tabular}

(no. RR470A; TaKaRa, Japan) according to the manufacturer's protocol. Oligonucleotides were designed and synthesized by Sangon Biotech Company. Real-time PCR was performed using the SYBR Green PCR Kit (no. RR820A; TaKaRa, Japan). The sequences of the primer pairs are shown in Table 1. Amplification was performed using the CFX96 Real-Time PCR detection system (Bio-Rad, USA). The cycling conditions were as follows: $95^{\circ} \mathrm{C}$ for $30 \mathrm{~s}$, followed by 40 cycles of $95^{\circ} \mathrm{C}$ for $5 \mathrm{~s}$ and $60^{\circ} \mathrm{C}$ for $30 \mathrm{~s}$. The mRNA levels were normalized to $\beta$-actin as a control. The data were presented as the relative fold change compared with the control.

\section{Western Blotting}

The cells were washed with phosphate-buffered saline and lysed with lysis buffer (Cell Signaling Technology, USA). The protein samples were separated by SDS-PAGE and transferred to PVDF membranes (Bio-Rad). The membranes were blocked with $5 \%$ non-fat milk and then were incubated overnight with primary antibodies against mPGES-1 (no. 160140; 1:500; Cayman, USA), fibronectin (no. ab45688; 1:1000; abCam, England), Collagen I (no. ab138492; 1:500; abCam), E-cadherin (no. 14472; 1:1000; Cell Signaling Technology), NLRP3 (no. ab263899; 1:1000; abCam), procaspase-1 and caspase-1 (no. ab179515; 1:500; abCam), and $\beta$-actin (no. 3700; 1:5000; Cell Signaling Technology) in TBS with $0.1 \%$ Tween 20. Next, the membranes were incubated with horseradish peroxidase (HRP)-conjugated anti-mouse IgG (no. 7076; 1:5000; Cell Signaling Technology) or anti-rabbit IgG antibodies (no. 7074; 1:5000; Cell Signaling Technology) for $1 \mathrm{~h}$ at room temperature. ECL chemiluminescence (Bio-Rad) was used to visualize the proteins using the ChemiDoc XRS + system (Bio-Rad).

\section{Statistical Analysis}

All the data were shown as mean \pm SEM. Statistical analysis was performed using SPSS 20.0 for Mac. Statistical differences were 
A

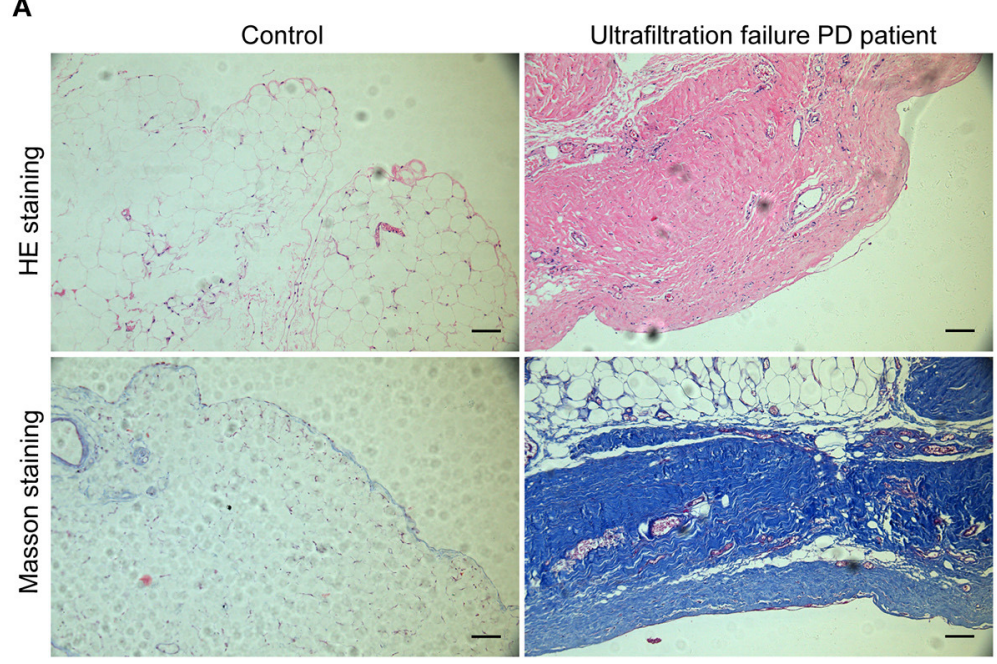

B

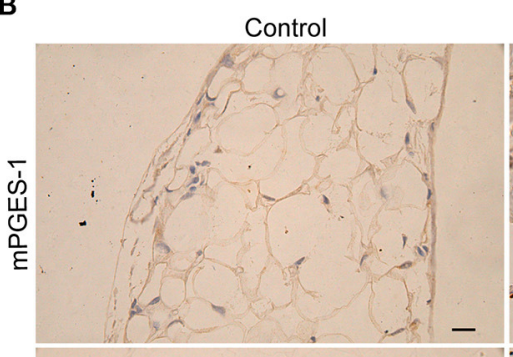

Ultrafiltration failure PD patient

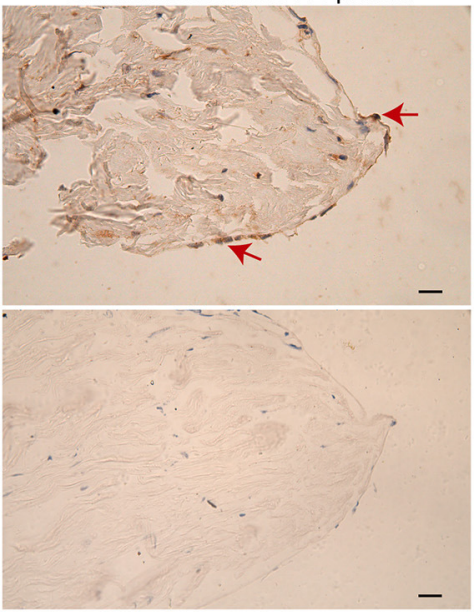

C

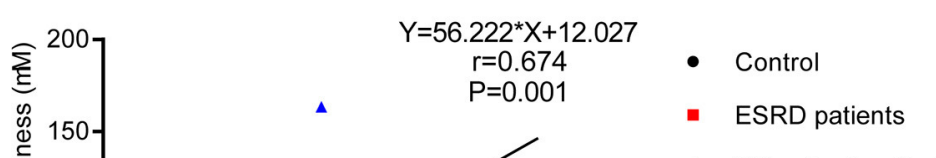

^ PD patients with UFF

FIGURE 1 | Enhanced expression of mPGES-1 in the peritoneal tissues of PD patients with ultrafiltration failure. (A) Representative HE and Masson staining in peritoneal tissues of inguinal hernia patients with normal renal function and PD patients with UFF. Magnification: 100x. Scale bar: $100 \mu \mathrm{m}$. (B) Representative image of immunohistochemical staining of mPGES-1 in the peritoneal tissues of inguinal hernia patients with normal renal function and PD patient with UFF. Analysis of mPGES-1 antibody specificity by replacing mPGES-1 antibody with isotype IgG. Magnification: 400x. Scale bar: 20 $\mu \mathrm{m}$. (C) Correlation between the expression of mPGES-1 in peritoneal tissues and the thickness of the peritoneum (Spearman's rank correlation analysis, $n=21$ ). Symbols of black dot, red square, and blue triangle represented control, ESRD patients, and PD patients with UFF, respectively. 
assessed by ANOVA followed by the LSD test for multiple groups. The correlations mentioned in this article were assessed by the Spearman's rank correlation coefficient. $P<0.05$ was deemed to indicate statistical significance.

\section{RESULTS}

\section{Enhanced mPGES-1 Expression in the Peritoneal Tissue of Ultrafiltration Failure PD Patients}

We initially studied the expression of mPGES-1 in the peritoneal tissue of inguinal hernia patients with normal renal function (control group) and PD patients with UFF. Loss of mesothelial cells and thickening of the submesothelial layer are important features of peritoneal fibrosis. HE staining and Masson's staining revealed that, compared with the peritoneal tissue of the control group, loss of the mesothelial cell monolayer and the thickness of the peritoneum significantly increased in PD patients with UFF (Figure 1A). Immunohistochemical analysis of the visceral peritoneum showed that mPGES-1 was expressed in residual mesothelial cells in ultrafiltration failure PD patients, while mPGES-1 was rarely expressed in inguinal hernia patients (Figure 1B). Furthermore, we analyzed the correlation between the expression of mPGES-1 in peritoneal tissue and thickness of the peritoneum. We collected 21 peritoneum samples from eight inguinal hernia patients, 8 ESRD patients and 5 PD patients with UFF. As shown in Figure 1C, the expression of mPGES1 in the peritoneum and thickness of the peritoneum showed a significant positive correlation. Data about the expression of mPGES-1 and thickness of peritoneum in individual patient were shown in Supplementary Material. These results indicate that the expression of mPGES-1 may be associated with peritoneal fibrosis.

\section{Increased PGE2 Secretion During PD Therapy}

PGE2 is the only downstream effector of mPGES-1. Next, we detected the secretion of PGE2 in PD fluid and investigated the relationship between PGE2 secretion and the clinical characteristics of PD patients. In total, $116 \mathrm{PD}$ patients were enrolled [mean age, $50.65 \pm 14.16$ (SD) years], with a median dialysis duration of 50 (maximum, 119) months. The mean PGE2 value was 256 (range, 10.00-472.10) $\mathrm{pg} / \mathrm{mL}$, and the mean ultrafiltration volume was 800 (range, 0-2,200) $\mathrm{mL} /$ day for all patients (Table 2). Considering that the concentration of PGE2 was affected by the patients' ultrafiltration volume, we analyzed the total secretion of PGE2 instead of the PGE2 concentration. Bivariate correlation analysis revealed that the total secretion of PGE2 was positively correlated with the PD duration, serum C-reactive protein level, and serum creatinine level but negatively correlated with the patients' daily residual urine volume (Figures 2A-D). These data demonstrate that the total secretion of PGE2 in PD fluid increases during PD therapy.
TABLE 2 | Patients' clinical characteristics

\begin{tabular}{lc}
\hline & $\boldsymbol{N}=\mathbf{1 1 6}$ \\
\hline Age (year) & $50.65 \pm 14.16$ \\
Male $(\%)$ & $66(56.8 \%)$ \\
BMI $\left(\mathrm{kg} / \mathrm{m}^{2}\right)$ & $21.80(19.90,24.20)$ \\
Dialysis duration $(\mathrm{month})$ & $50.00(39.25,61.75)$ \\
Dialysate PGE2 $(\mathrm{pg} / \mathrm{mL})$ & $256.18 \pm 101.95$ \\
Ultrafiltration volume $(\mathrm{mL} /$ day $)$ & $800.00(432.50,1096.25)$ \\
Mean arterial pressure $(\mathrm{mmHg})$ & $99.67(92.33,105.50)$ \\
Serum C-reactive protein $(\mathrm{mg} / \mathrm{L})$ & $2.34(0.99,5.08)$ \\
Serum hemoglobin $(\mathrm{g} / \mathrm{L})$ & $110.00(95.50,120.00)$ \\
Serum creatinine $(\mathrm{umol} / \mathrm{L})$ & $1089.02 \pm 343.35$ \\
Albumin-corrected calcium $(\mathrm{mmol} / \mathrm{L})$ & $2.30(2.15,2.46)$ \\
Total Kt/ $\mathrm{N}$ & $1.98(1.72,2.35)$ \\
Residual urine volume $(\mathrm{mL} / \mathrm{day})$ & $200.00(0.00,537.50)$
\end{tabular}

\section{Induction of mPGES-1 Expression by High Glucose in RPMCs}

Next, we detected the expression change of mPGES-1 in RPMCs after high glucose damage. First, the expression levels of mPGES1, mPGES-2, and cPGES in RPMCs under high glucose were detected. After treating RPMCs with glucose at the concentration of $138 \mathrm{mmol} / \mathrm{L}$ for $0,24,48$, and $72 \mathrm{~h}$, the mRNA expression of mPGES-1, but not those of mPGES-2 and cPGES, was significantly up-regulated as determined by quantitative RTPCR (Figure 3A). Western blotting showed that the expression level of mPGES-1 was significantly increased in high-glucoseinduced RPMCs (Figures 3B,C), and ELISA showed that the secretion of PGE2 in supernatants was markedly elevated in a time-dependent manner in RPMCs treated with high glucose (Figure 3D).

\section{Inhibition of mPGES-1 Ameliorates High-Glucose-Induced Synthesis of Extracellular Matrix Proteins in RPMCs}

To further analyze the contribution of mPGES-1/PGE2 signaling to peritoneal fibrosis, we analyzed its effect on the synthesis of extracellular matrix proteins in RPMCs after transfection with mPGES-1 shRNA or control shRNA. The PGE2 release in supernatants was significantly elevated in response to high-glucose treatment (Figure 4A). This increase in PGE2 was abolished by silencing mPGES-1 (Figure 4A). As shown in Figures 4B,C, mPGES-1 shRNA significantly reduced the expression of mPGES-1 in the high-glucose condition. By application of mPGES-1 shRNA, high-glucoseinduced upregulation of fibronectin $(\mathrm{FN})$ and collagen I was significantly blunted (Figures 4B,D,E), while high-glucoseinduced downregulation of E-cadherin was significantly increased (Figures 4B,F). 

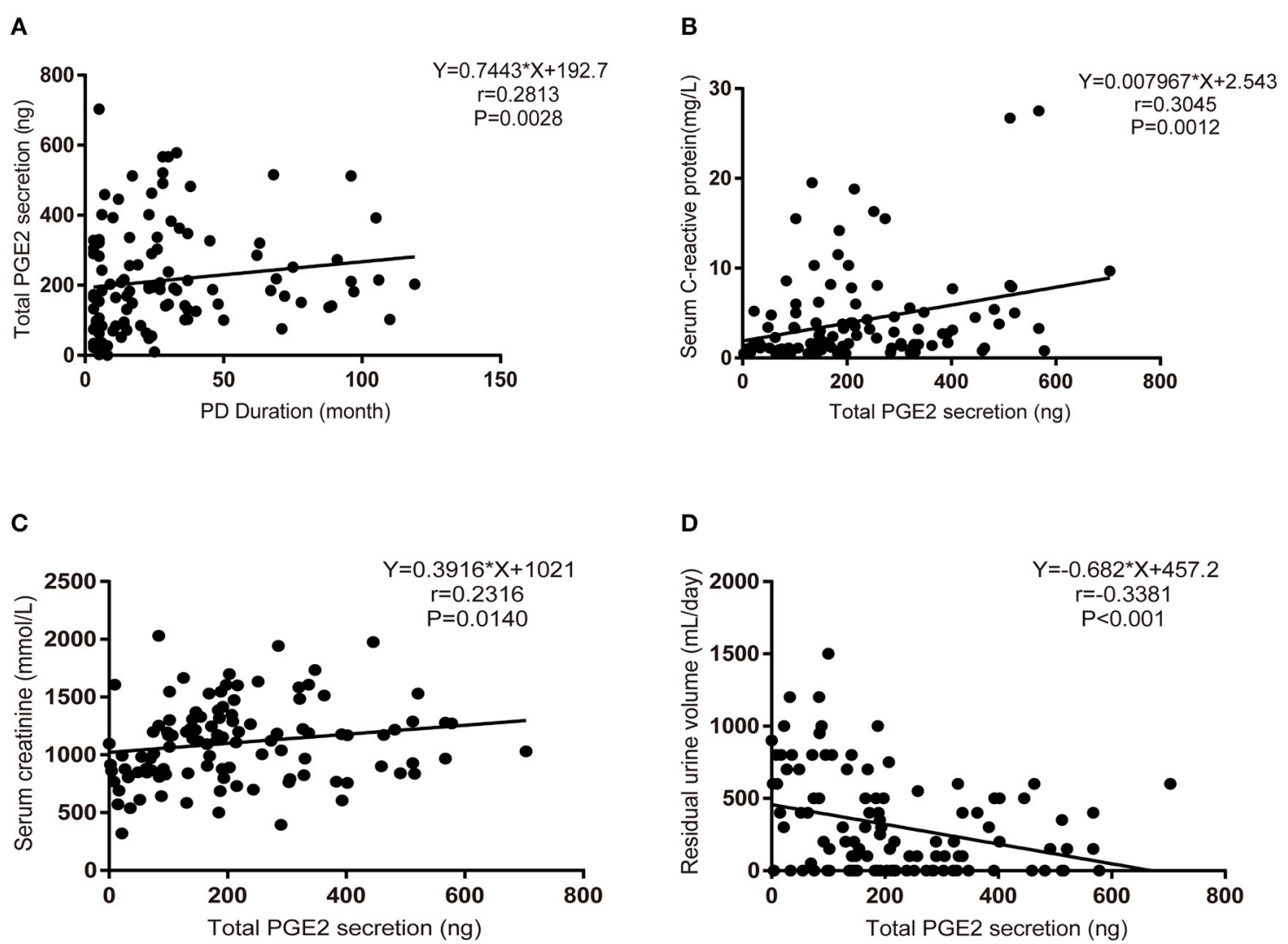

FIGURE 2 | Increased PGE2 secretion during PD therapy. (A) Bivariate correlation analysis between the total secretion of PGE2 and the PD duration in incident PD patients (Spearman's rank correlation analysis, $n=116)$. (B) Bivariate correlation analysis between the total secretion of PGE2 and serum C-reactive protein levels in incident PD patients (Spearman's rank correlation analysis, $n=116)$. (C) Bivariate correlation analysis between the total secretion of PGE2 and serum creatinine levels in incident PD patients (Spearman's rank correlation analysis, $n=116$ ). (D) Bivariate correlation analysis between the total secretion of PGE2 and daily residual urine volume levels in incident PD patients (Spearman's rank correlation analysis, $n=116$ ).

\section{Inhibition of mPGES-1 Blunts High-Glucose-Induced Activation of the NLRP3 Inflammasome}

We next investigated how mPGES-1 mediates extracellular matrix protein synthesis in RPMCs. High-glucose-upregulated mRNA levels of NLRP3, activated caspase-1, and IL-18 were significantly blunted in RPMCs transfected with mPGES1 shRNA (Figures 5A-C). After treating RPMCs with high glucose, the expression of NLRP3 was induced (Figure 5D). Western blotting further confirmed the induction of NLRP3 and activated caspase- 1 at the protein levels, a phenomenon that was remarkably reduced by mPGES- 1 shRNA (Figures $5 \mathrm{D}-\mathbf{H}$ ). There was no significantly change of procaspase-1 protein in RPMCs treated with mPGES-1 shRNA.

\section{Inhibition of NLRP3 by siRNA Abrogates High-Glucose-Induced Synthesis of Extracellular Matrix Proteins in RPMCs}

Our data suggest that mPGES-1 can mediate the activation of the NLRP3 inflammasome. We next explored whether the NLRP3 inflammasome is involved in high-glucose-induced synthesis of extracellular matrix proteins in RPMCs. NLRP3 siRNA was applied to inhibit the expression of NLRP3 in RPMCs treated with high glucose. Western blotting confirmed the induction of FN and collagen-I protein levels under high glucose was significantly reduced by NLRP3 siRNA treatment, and the reduction of E-cadherin protein levels under high glucose was significantly reversed (Figures 6A-E).

\section{Positive Correlation Between the Secretion of PGE2 and IL-1 $\beta$ or IL-18 in PD Fluid}

IL-1 $\beta$ and IL-18 are the major products of the activation of the NLRP3 inflammasome. We next detected the secretion of IL-1 $\beta$ and IL-18 in the dialysate and explored the relationship between the secretion of PGE2 and IL-1 $\beta$ or IL-18 in PD fluid. Fifty-three patients were enrolled [mean age, $46.43 \pm 13.30$ (SD) years], and their mean concentrations of IL- $1 \beta$ and IL-18 were 69.12 and $107.48 \mathrm{pg} / \mathrm{L}$, respectively. IL-1 $\beta$ and IL-18 were corrected by ultrafiltration volumes because of the difference in the daily ultrafiltration volume in PD patients. As shown in Figure 7, the total secretion of PGE2 was positively correlated with the total secretion of IL-1 $\beta(r=0.7836 ; P<0.001)$ (Figure 7A) and 
A

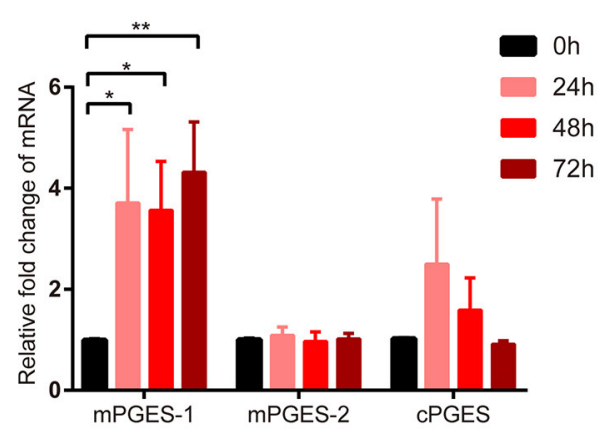

C

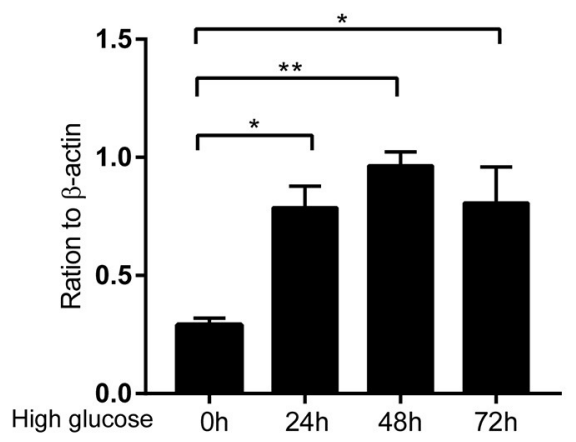

B

High glucose(h) $\underline{0 \quad 24 \quad 4872} \underline{0 \quad 244872} \underline{0 \quad 244872}$

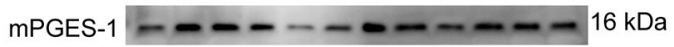

$\beta$-actin $-0-0-53 \mathrm{kDa}$

D

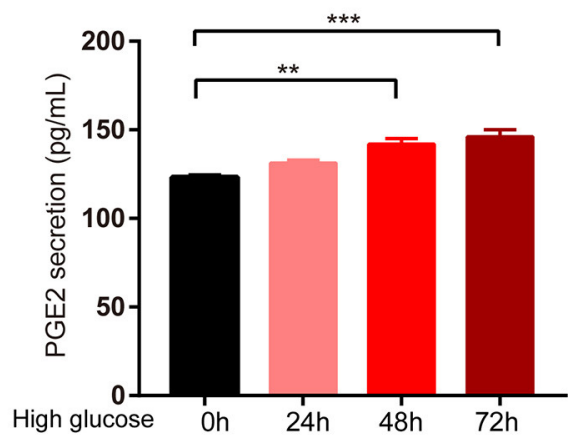

FIGURE 3 | Induction of mPGES-1 expression by high glucose in RPMCs. (A) Real-time quantitative PCR analysis of mPGES-1, mPGES-2, and cPGES levels in RPMCs in response to high glucose $(138 \mathrm{mmol} / \mathrm{L})$ for the indicated times. (B) RPMCs were incubated with high glucose $(138 \mathrm{mmol} / \mathrm{L})$ for the indicated times, and western blotting detected the expression of mPGES-1. (C) Quantitative analysis of mPGES-1 expression in RPMCs treated with high glucose (138 mmol/L) for the indicated times. (D) RPMCs were treated with high glucose (138 mmol/L) for the indicated times, and ELISA was performed for PGE2 in the cell culture medium. The data are represented as the mean \pm SEM. ${ }^{\star} P<0.05$, ${ }^{* \star} P<0.01$, and ${ }^{\star \star \star} P<0.001$.

total secretion of IL-18 $(r=0.8366 ; P<0.001)$ (Figure 7B) in the dialysate.

\section{DISCUSSION}

In this study, we combined clinical and functional data in humans and RPMCs to demonstrate that mPGES-1/PGE2 signaling is activated in PD patients with UFF and the expression of mPGES-1 in the peritoneum is positively correlated with peritoneal fibrosis. Inhibition of mPGES-1 in vitro ameliorated high-glucose-induced synthesis of extracellular matrix proteins in RPMCs through blunting the activation of the NLRP3 inflammasome. Compared with traditional NSAIDs, targeting mPGES-1 may be a safer and more effective alternative to control acute inflammation, and mPGES-1 inhibitors are expected to be a new generation of anti-inflammatory drugs $(22,23)$. Our data revealed the perspectives of targeting mPGES-1 to attenuate $\mathrm{PD}$-associated peritoneal fibrosis.

Previous studies have demonstrated that the expression of mPGES-1 is increased in inflammatory diseases, including colitis, arthritis, and gastritis (24-26). Our present study found that mPGES-1 is activated in the peritoneum of PD patients with UFF, and the expression was also increased in RPMCs treated with high glucose. The results from this study revealed that, in addition to the acute phase of diseases, mPGES- 1 is still activated under sustained pathological stimulation, a finding that was consistent with the induction of mPGES-1 in peripheral blood mononuclear cells from hypertensive patients, kidney tissues of the unilateral ureteral obstruction mouse model and renal proximal tubular cells treated with albumin (27-29). Additionally, in our study, a positive correlation was found between the expression of mPGES-1 in the peritoneum and degree of peritoneal fibrosis. Thus, mPGES-1 maybe involved in the progression of peritoneal fibrosis. The results from our in vitro study further support that mPGES-1 plays an important role in the synthesis of fibrotic proteins in RPMCs, a pivotal event during peritoneal fibrosis. However, the role of mPGES1 in fibrosis is controversial according to previous studies. In the unilateral ureteral obstruction mouse model, mPGES-1 exerts a potentially protective effect against renal fibrosis, while inhibition of mPGES-1 provides a viable method to alleviate the development of bleomycin-induced skin fibrogenesis $(27,30)$. These different effects on fibrosis may be attributed to different models of disease and tissue-specific mechanisms.

Peritoneal fibrosis during PD is a chronic inflammationdriven process, and PGE2 is a major proinflammatory mediator 
A

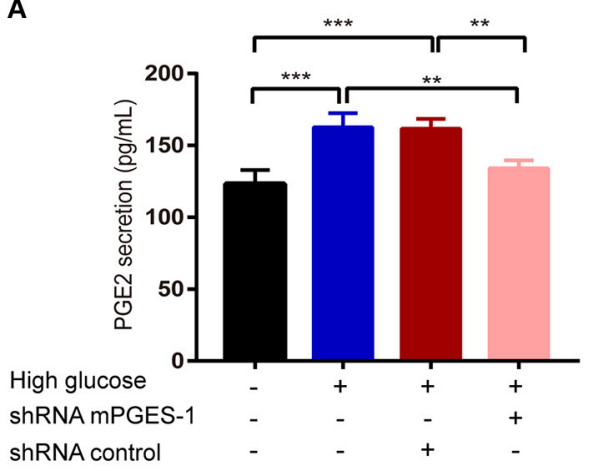

C

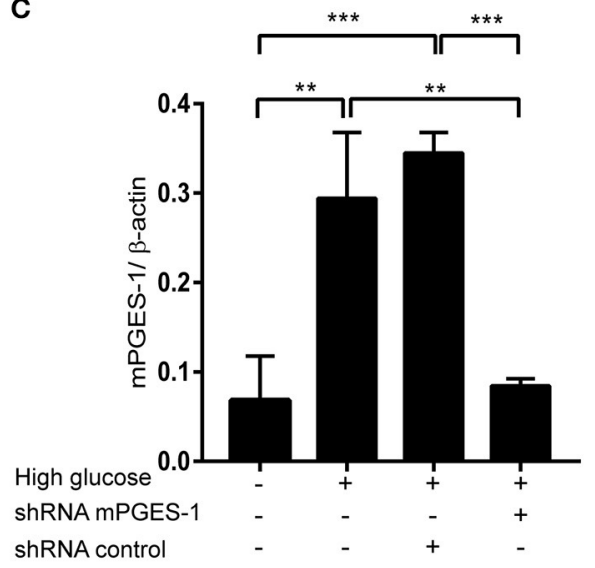

E

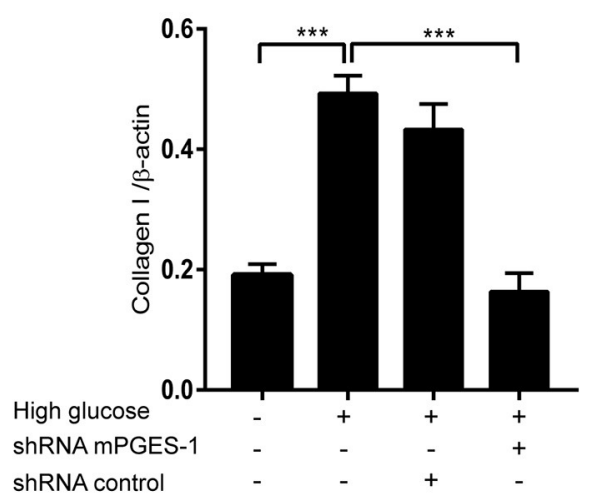

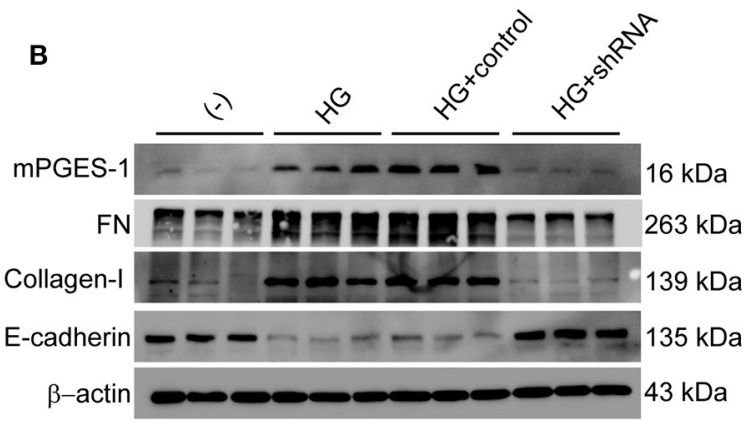

D

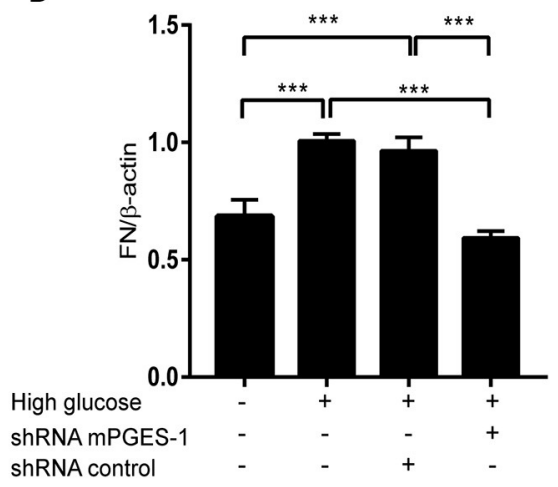

$\mathbf{F}$

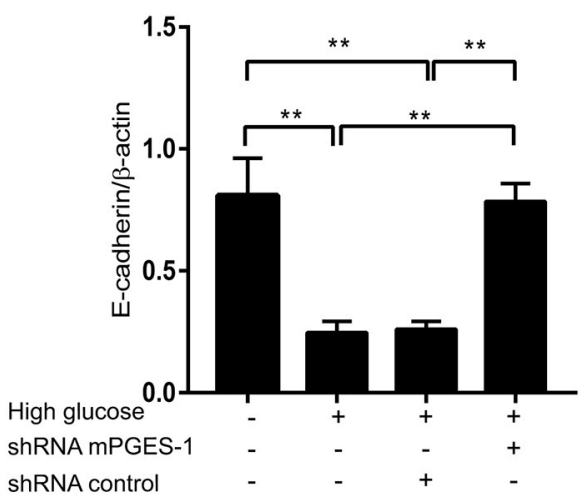

FIGURE 4 | Inhibition of mPGES-1 ameliorates high-glucose-induced synthesis of extracellular matrix proteins in RPMCs. (A) RPMCs were treated with mPGES-1 shRNA plasmid or control shRNA plasmid, followed by incubation with high glucose for $48 \mathrm{~h}$. ELISA of PGE2 in cell culture medium in RPMCs treated with normal glucose, high glucose, high glucose plus shRNA control or high glucose plus mPGES-1 shRNA. (B) RPMCs were transfected with the mPGES-1 shRNA plasmid or control shRNA plasmid, followed by incubation with high glucose for $48 \mathrm{~h}$. Western blotting confirmed the expression of mPGES-1, FN, Collagen-I, and E-cadherin. Quantitative analyses of mPGES-1, FN, Collagen-I, and E-cadherin were displayed in the (C-F). The data are represented as the mean \pm SEM. ${ }^{* \star} P<0.01$, ${ }^{\star \star \star} P<$ 0.001 .

that is up-regulated during inflammation. Considering PGE2 is the terminal product of mPGES-1, we analyzed the relationship between the secretion of PGE2 in the dialysate and clinical characteristics of the PD patients. With a prolonged $\mathrm{PD}$ duration, the degree of peritoneal fibrosis became more severe. In our study, the expression of mPGES-1 increased with the 
A

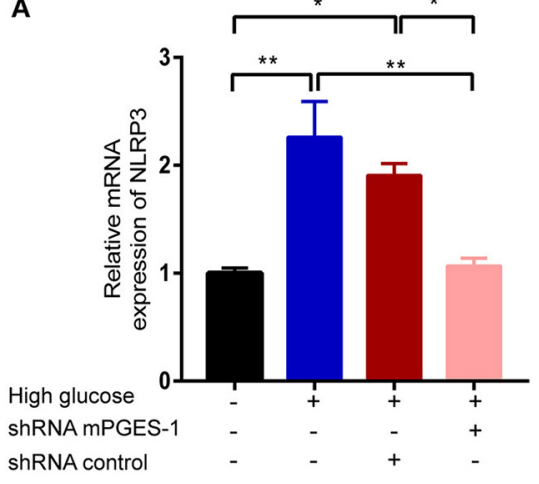

C

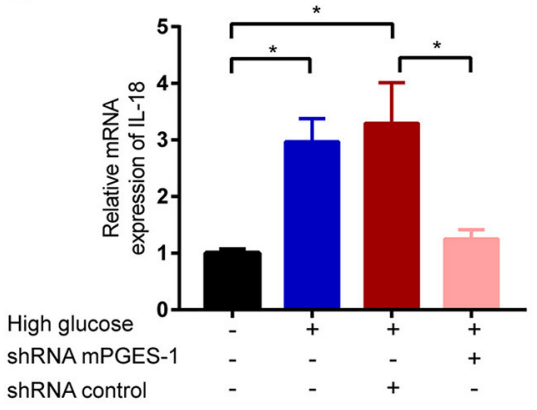

E

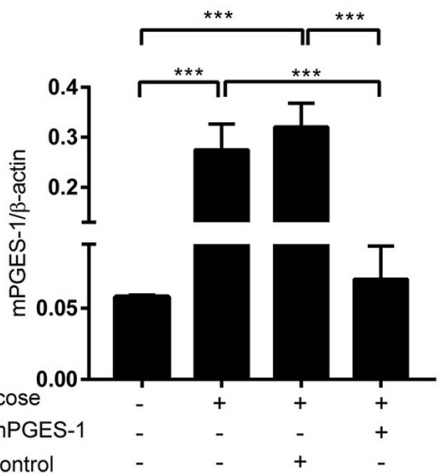

G

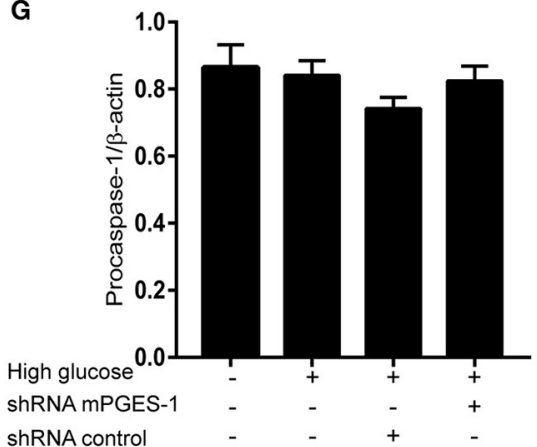

B

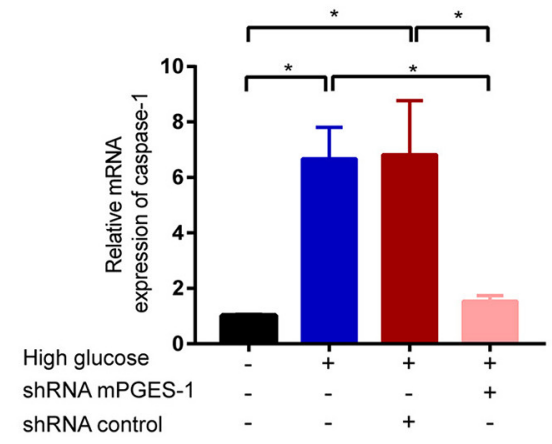

D
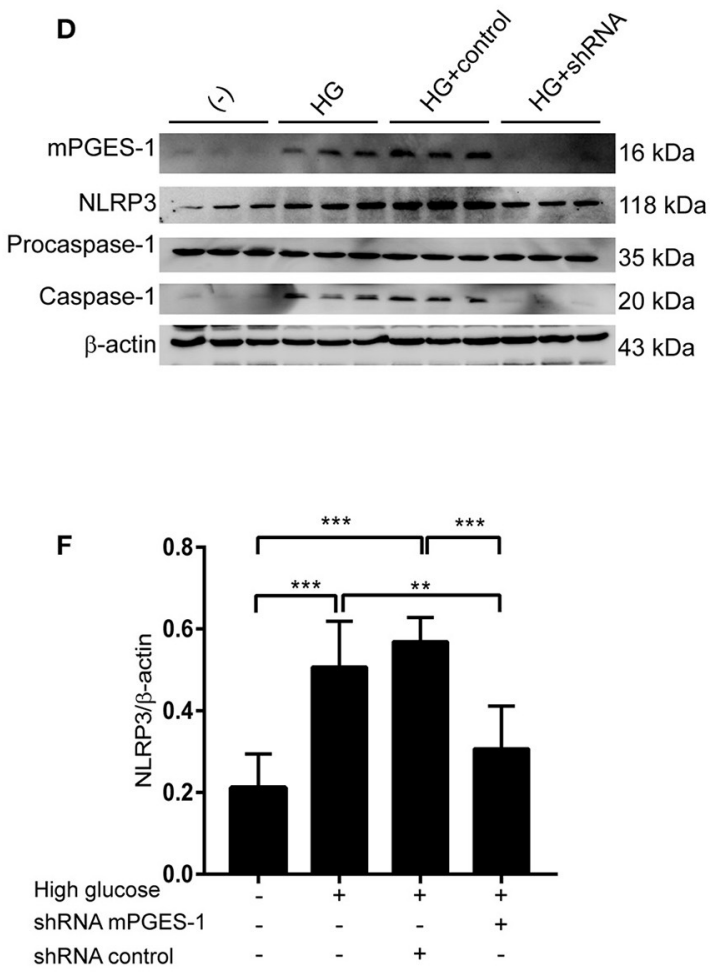

H

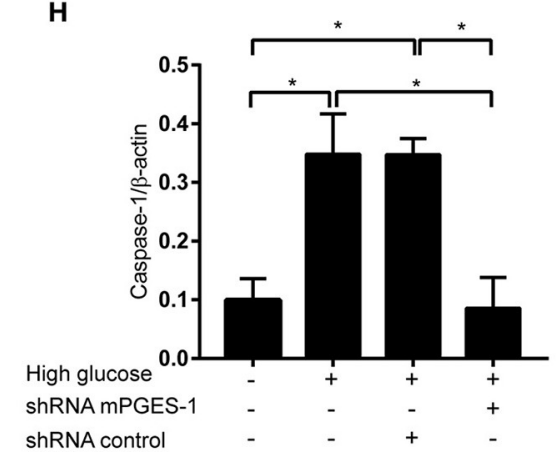

FIGURE 5 | Inhibition of mPGES-1 blunts high-glucose-induced activation of the NLRP3 inflammasome. (A-C) RPMCs were transfected with the mPGES-1 shRNA plasmid or control shRNA plasmid, followed by incubation with high glucose for $24 \mathrm{~h}$. Real-time quantitative PCR analysis was used to detect the mRNA levels of NLRP3 (A), caspase-1 (B), and IL-18 (C). (D-H) RPMCs were transfected with the mPGES-1 shRNA plasmid or control shRNA plasmid, followed by incubation with high glucose for $48 \mathrm{~h}$. Western blotting confirmed the expression of mPGES-1, NLRP3, procaspase-1, and caspase-1. Quantitative analyses of mPGES-1 (E), NLRP3 (F), procaspase-1 (G), and caspase-1 (H) expression were displayed. The data are represented as the mean $\pm \mathrm{SEM}$. ${ }^{\star} P<0.05$, ${ }^{\star \star} P<0.01,{ }^{\star \star \star} P<0.001$. 


\section{A}

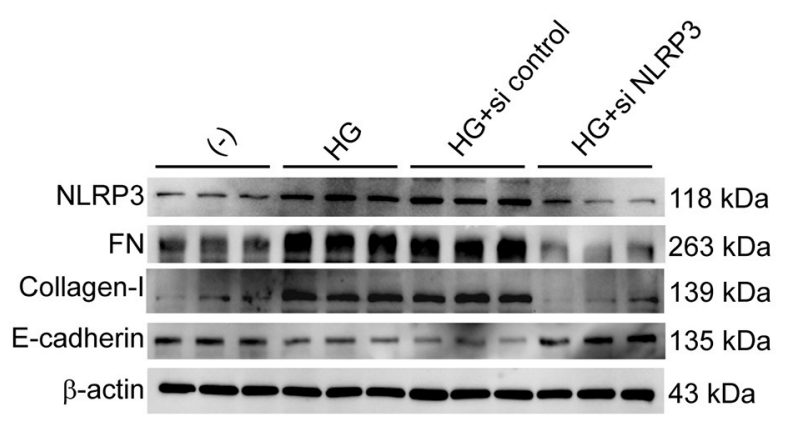

C

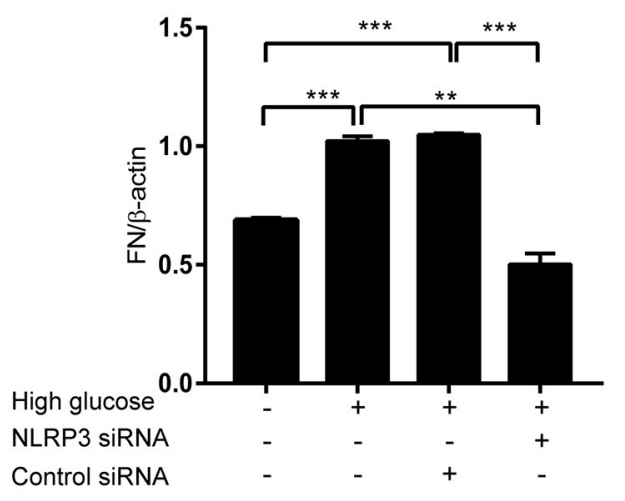

E

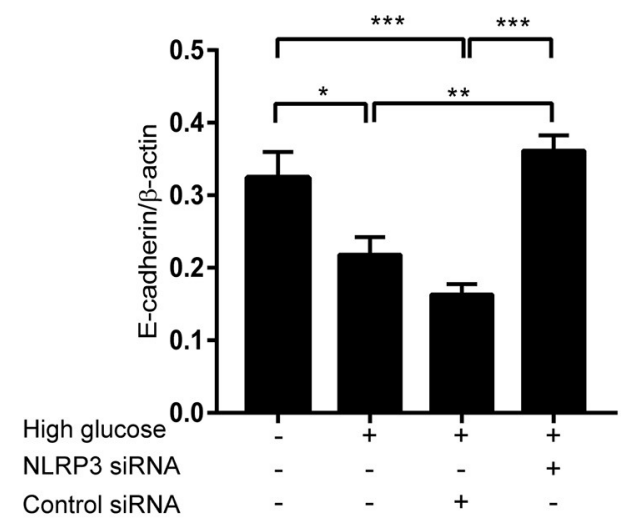

B

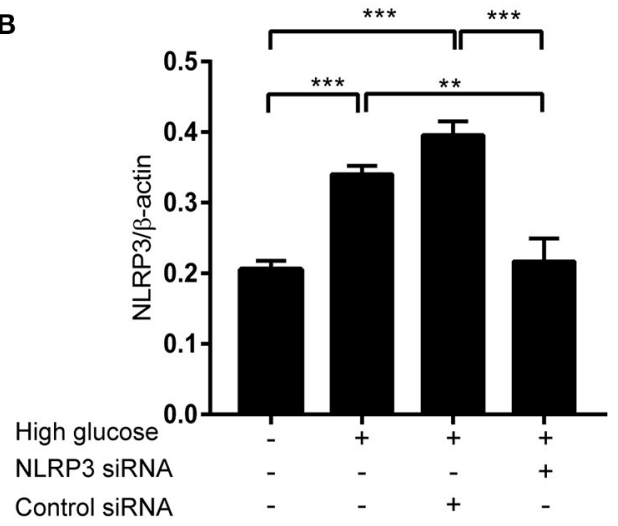

D

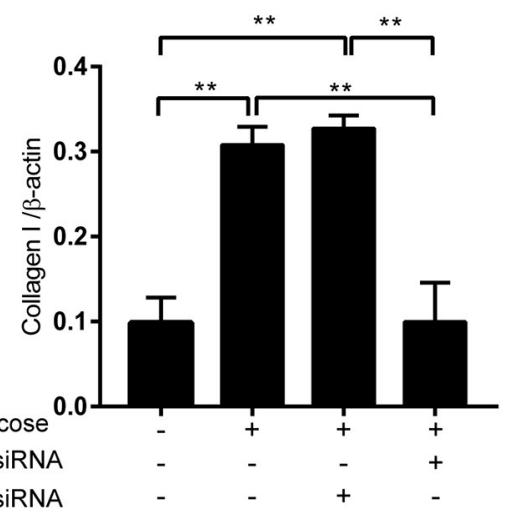

FIGURE 6 | Inhibition of NLRP3 by siRNA abrogates high-glucose-induced synthesis of extracellular matrix proteins in RBMCs. (A) RBMCs were treated with NLRP3 siRNA or control siRNA, followed by incubation with high glucose for $48 \mathrm{~h}$. Western blotting confirmed the expression of NLRP3, FN, Collagen-I, and E-cadherin. Quantitative analyses of NLRP3, FN, Collagen-I, and E-cadherin were displayed in the (B-E). The data are represented as the mean \pm SEM. ${ }^{\star} P<0.05$, ${ }^{* *} P<0.01$, ${ }^{\star \star \star} P<0.001$

aggravation of peritoneal fibrosis, and the secretion of PGE2 in the peritoneum of our $\mathrm{PD}$ patients was positively correlated with the PD duration. Additionally, PGE2 was negatively correlated with the residual urine volume and positively correlated with the serum creatinine level in our study. The major functions of the peritoneum during PD therapy include ultrafiltration of liquid and transport of small soluble molecules. A decreased residual urine volume and higher serum creatinine levels can represent worse peritoneal membrane function. Additionally, with a prolonged PD duration, the residual urine volume decreases, a finding that was also demonstrated in our study. Single isolated episodes of peritonitis have no significant effect on peritoneal 
A

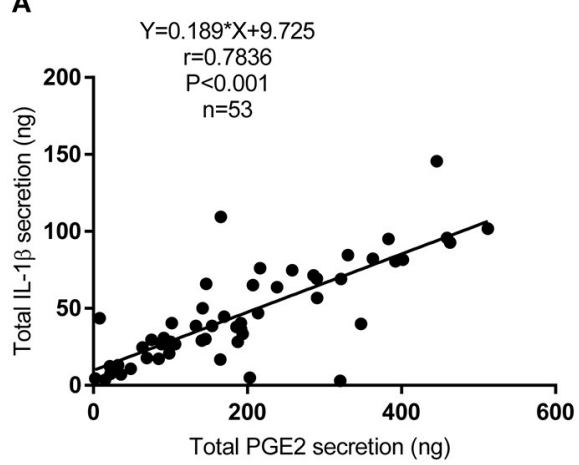

B

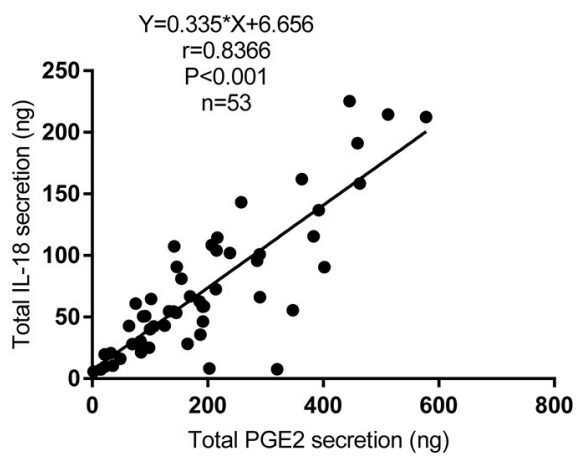

FIGURE 7 | Correlation between the secretion of PGE2 and IL-1 $\beta$ or IL-18 in PD fluid. (A) Bivariate correlation analysis between the total secretion of PGE2 and IL-1 $\beta$ in incident PD patients' dialysate (Spearman's rank correlation analysis, $n=53$ ). (B) Bivariate correlation analysis between the total secretion of PGE2 and IL-18 in incident PD patients' dialysate (Spearman's rank correlation analysis, $n=53$ ). creatinine transport (31); however, the results from the Global Fluid Study showed that intraperitoneal inflammation is the most important determinant of peritoneal small solute transport in incident PD patients (32). However, we found no statistical difference in the correlation between the intraperitoneal PGE2 levels and transport of small soluble molecules, partly due to the limitation of the enrollment number. More incident PD patients need to be recruited to further explore the relationship between the intraperitoneal PGE2 levels and small soluble molecule transport.

The results of our study showed a significant correlation between secretion of PGE2 and serum C-reactive protein. Serum C-reactive protein is a critical systemic biomarker of inflammation. A previous study indicated that during the first year of therapy, intraperitoneal and systemic inflammation increased in patients with PD treatment (33). Our results showed that, in PD patients, local intraperitoneal inflammation is associated with systemic inflammation. The results of a retrospective cohort study that recruited $402 \mathrm{PD}$ patients demonstrated that the serum C-reactive protein level is an independent predictor of technique failure (34), suggesting that systemic inflammation is associated with technique survival.
mPGES-1/PGE2 plays a critical role in the progression of multiple diseases via proinflammatory mechanisms $(28,35$, 36). We explored the effect between mPGES-1/PGE2 signaling and the NLRP3 inflammasome in high-glucose-stimulated RPMCs. Recently, increasing evidence has demonstrated the influence of mPGES-1/PGE2 on NLRP3 inflammation in different cell types. Blockade of COX-2/mPGES- 1 could inhibit macrophage M1 polarization and NLRP3 inflammation activation in response to monosodium urate crystals (37). Additionally, PGE2 activates NLRP3 inflammation in endothelial cells to promote diabetic retinopathy (38). Furthermore, high-glucose-based PD solutions trigger NLRP3 inflammation activation in human peritoneal mesothelial cells (39), and angiotensin- (1-7) attenuated angiotensin-induced epithelialmesenchymal transition in hepatocytes through inhibiting the NAPDH oxidase-derived, hydrogen peroxide-activated NLRP3 inflammation (40). In our study, we found that inhibiting NLRP3 in high-glucose-stimulated RPMCs attenuates the expression of extracellular matrix proteins.

Although this study demonstrated the positive relationship between mPGES-1/PGE2 and PD-related peritoneal fibrosis and the beneficial effect of the inhibition of mPGES-1 on the production of extracellular matrix proteins in RPMCs, the therapeutic effect targeting $\mathrm{mPGES}-1$ were not evaluated in the PD animal model. Besides, mPGES-1/PGE2 exerts the functions through its downstream prostaglandin receptors. In this research work, we did not investigate the contribution of prostaglandin E2 receptors during the NLRP3 inflammasome activation and peritoneal fibrosis. Further studies are required to be performed to address these questions.

In summary, the present study demonstrated that mPGES1/PGE2 signaling is activated during PD therapy, and the intraperitoneal PGE2 levels are associated with systemic inflammation and the PD duration. Furthermore, mPGES-1 contributes to increase the synthesis of extracellular matrix proteins of RPMCs by activating NLRP3 inflammation. Targeting mPGES-1 may attenuate the progression of peritoneal fibrosis during PD treatment.

\section{DATA AVAILABILITY STATEMENT}

The raw data supporting the conclusions of this article will be made available by the authors, without undue reservation.

\section{ETHICS STATEMENT}

The studies involving human participants were reviewed and approved by Ethical Review Board of Shunde Hospital of Southern Medical University. The patients/participants provided their written informed consent to participate in this study.

\section{AUTHOR CONTRIBUTIONS}

ZJ and XD coordinated and oversaw the study. QL, QH, QZ, LG, LS, BR, and YJ collected samples, performed experiments, and 
analyzed data. QL, ZJ, and XD wrote the manuscript. All authors contributed to the article and approved the submitted version.

\section{FUNDING}

This work was supported by grants from the National Natural Science Foundation for Yong Scholars of China (Grant No. 81800674), Natural Science Foundation of Guangdong Province

\section{REFERENCES}

1. Liu ZH. Nephrology in China. Nat Rev Nephrol. (2013) 9:5238. doi: 10.1038/nrneph.2013.146

2. Fusshoeller A. Histomorphological and functional changes of the peritoneal membrane during long-term peritoneal dialysis. Pediatr Nephrol. (2008) 23:19-25. doi: 10.1007/s00467-007-0541-z

3. Aroeira LS, Aguilera A, Sanchez-Tomero JA, Bajo MA, del Peso G, JimenezHeffernan JA, et al. Epithelial to mesenchymal transition and peritoneal membrane failure in peritoneal dialysis patients: pathologic significance and potential therapeutic interventions. J Am Soc Nephrol. (2007) 18:200413. doi: 10.1681/ASN.2006111292

4. Davies SJ, Mushahar L, Yu Z, Lambie M. Determinants of peritoneal membrane function over time. Semin Nephrol. (2011) 31:172-82. doi: 10.1016/j.semnephrol.2011.01.006

5. Yung S, Chan TM. Pathophysiological changes to the peritoneal membrane during PD-related peritonitis: the role of mesothelial cells. Mediat Inflamm. (2012) 2012:484167. doi: 10.1155/2012/484167

6. Morelle J, Sow A, Hautem N, Bouzin C, Crott R, Devuyst O, et al. Interstitial fibrosis restricts osmotic water transport in encapsulating peritoneal sclerosis. J Am Soc Nephrol. (2015) 26:2521-33. doi: 10.1681/ASN.2014090939

7. Lai KN, Tang SC, Leung JC. Mediators of inflammation and fibrosis. Perit Dial Int. (2007) 27 (Suppl. 2):S65-71. doi: 10.1177/089686080702702s12

8. Rosengren BI, Sagstad SJ, Karlsen TV, Wiig H. Isolation of interstitial fluid and demonstration of local proinflammatory cytokine production and increased absorptive gradient in chronic peritoneal dialysis. Am J Physiol Renal Physiol. (2013) 304:F198-206. doi: 10.1152/ajprenal.00293.2012

9. Lambie MR, Chess J, Summers AM, Williams PF, Topley N, Davies SJ. Peritoneal inflammation precedes encapsulating peritoneal sclerosis: results from the GLOBAL fluid study. Nephrol Dial Transplant. (2016) 31:4806. doi: $10.1093 / \mathrm{ndt} / \mathrm{gfv} 440$

10. Choi SY, Ryu HM, Choi JY, Cho JH, Kim CD, Kim YL, et al. The role of Toll-like receptor 4 in high-glucose-induced inflammatory and fibrosis markers in human peritoneal mesothelial cells. Int Urol Nephrol. (2017) 49:171-81. doi: 10.1007/s11255-016-1430-9

11. Chu Y, Wang Y, Zheng Z, Lin Y, He R, Liu J, et al. Proinflammatory effect of high glucose concentrations on HMrSV5 cells via the autocrine effect of HMGB1. Front Physiol. (2017) 8:762. doi: 10.3389/fphys.2017.00762

12. Strippoli R, Moreno-Vicente R, Battistelli C, Cicchini C, Noce V, Amicone L, et al. Molecular mechanisms underlying peritoneal EMT and fibrosis. Stem Cells Int. (2016) 2016:3543678. doi: 10.1155/2016/3543678

13. Chang $\mathrm{HH}$, Meuillet EJ. Identification and development of mPGES1 inhibitors: where we are at? Future Med Chem. (2011) 3:190934. doi: 10.4155/fmc.11.136

14. Wang M, Song WL, Cheng Y, Fitzgerald GA. Microsomal prostaglandin E synthase-1 inhibition in cardiovascular inflammatory disease. J Intern Med. (2008) 263:500-5. doi: 10.1111/j.1365-2796.2008.01938.x

15. Wang $M$, Ihida-Stansbury K, Kothapalli D, Tamby MC, Yu $\mathrm{Z}$, Chen L, et al. Microsomal prostaglandin e2 synthase-1 modulates the response to vascular injury. Circulation. (2011) 123:631-9. doi: 10.1161/CIRCULATIONAHA.110.973685

16. Kamei D, Yamakawa K, Takegoshi Y, Mikami-Nakanishi M, Nakatani Y, OhIshi S, et al. Reduced pain hypersensitivity and inflammation in mice lacking microsomal prostaglandin e synthase-1. J Biol Chem. (2004) 279:3368495. doi: 10.1074/jbc.M400199200
(Grant No. 2018A030310428), and China Postdoctoral Science Foundation funded project (Grant No. 2019M663006).

\section{SUPPLEMENTARY MATERIAL}

The Supplementary Material for this article can be found online at: https://www.frontiersin.org/articles/10.3389/fmed. 2021.675363/full\#supplementary-material

17. Sitter T, Haslinger B, Mandl S, Fricke H, Held E, Sellmayer A. High glucose increases prostaglandin E2 synthesis in human peritoneal mesothelial cells: role of hyperosmolarity. J Am Soc Nephrol. (1998) 9:2005-12.

18. Hautem N, Morelle J, Sow A, Corbet C, Feron O, Goffin E, et al. The NLRP3 inflammasome has a critical role in peritoneal dialysis-related peritonitis. J Am Soc Nephrol. (2017) 28:2038-52. doi: 10.1681/ASN.2016070729

19. Wu J, Liu B, Mao W, Feng S, Yao Y, Bai F, et al. Prostaglandin E2 regulates activation of mouse peritoneal macrophages by staphylococcus aureus through toll-like receptor 2, toll-like receptor 4, and NLRP3 inflammasome signaling. J Innate Immun. (2020) 12:154-69. doi: 10.1159/000499604

20. Teitelbaum I. Ultrafiltration failure in peritoneal dialysis: a pathophysiologic approach. Blood Purif. (2015) 39:70-3. doi: 10.1159/000368972

21. Ramos-Vara JA. Principles and methods of immunohistochemistry. Methods Mol Biol. (2011) 691:83-96. doi: 10.1007/978-1-60761-849-2_5

22. Martin EM, Jones SL. Inhibition of microsomal prostaglandin Esynthase-1 (mPGES-1) selectively suppresses PGE2 in an in vitro equine inflammation model. Vet Immunol Immunopathol. (2017) 192:33-40. doi: 10.1016/j.vetimm.2017.09.008

23. Ozen G, Gomez I, Daci A, Deschildre C, Boubaya L, Teskin O, et al. Inhibition of microsomal PGE synthase-1 reduces human vascular tone by increasing PGI2 : a safer alternative to COX-2 inhibition. Br J Pharmacol. (2017) 174:4087-98. doi: 10.1111/bph.13939

24. Gudis K, Tatsuguchi A, Wada K, Futagami S, Nagata K, Hiratsuka T, et al. Microsomal prostaglandin E synthase (mPGES)-1, mPGES-2 and cytosolic PGES expression in human gastritis and gastric ulcer tissue. Lab Invest. (2005) 85:225-36. doi: 10.1038/labinvest.3700200

25. Zhou H, Liu JX, Luo JF, Cheng CS, Leung EL, Li Y, et al. Suppressing mPGES1 expression by sinomenine ameliorates inflammation and arthritis. Biochem Pharmacol. (2017) 142:133-44. doi: 10.1016/j.bcp.2017.07.010

26. Maseda D, Banerjee A, Johnson EM, Washington MK, Kim H, Lau $\mathrm{KS}$, et al. mPGES-1-mediated production of PGE2 and EP4 receptor sensing regulate $\mathrm{T}$ cell colonic inflammation. Front Immunol. (2018) 9:2954. doi: 10.3389/fimmu.2018.02954

27. Luo R, Kakizoe Y, Wang F, Fan X, Hu S, Yang T, et al. Deficiency of mPGES-1 exacerbates renal fibrosis and inflammation in mice with unilateral ureteral obstruction. Am J Physiol Renal Physiol. (2017) 312:F121F33. doi: 10.1152/ajprenal.00231.2016

28. Avendano MS, Garcia-Redondo AB, Zalba G, Gonzalez-Amor M, Aguado A, Martinez-Revelles S, et al. mPGES-1 (Microsomal Prostaglandin E Synthase-1) mediates vascular dysfunction in hypertension through oxidative stress. Hypertension. (2018) 72:492-502. doi: 10.1161/HYPERTENSIONAHA.118.10833

29. Zhuang Y, Wang C, Wu C, Ding D, Zhao F, Hu C, et al. Mitochondrial oxidative stress activates COX-2/mPGES-1/PGE2 cascade induced by albumin in renal proximal tubular cells. Oncotarget. (2018) 9:923545. doi: 10.18632/oncotarget.24187

30. McCann MR, Monemdjou R, Ghassemi-Kakroodi P, Fahmi H, Perez G, Liu $\mathrm{S}$, et al. mPGES-1 null mice are resistant to bleomycin-induced skin fibrosis. Arthritis Res Ther. (2011) 13:R6. doi: 10.1186/ar3226

31. Davies SJ, Bryan J, Phillips L, Russell GI. Longitudinal changes in peritoneal kinetics: the effects of peritoneal dialysis and peritonitis. Nephrol Dial Transpl. (1996) 11:498-506. doi: 10.1093/oxfordjournals.ndt.a027318

32. Lambie M, Chess J, Donovan KL, Kim YL, Do JY, Lee HB, et al. Independent effects of systemic and peritoneal inflammation on peritoneal dialysis survival. J Am Soc Nephrol. (2013) 24:2071-80. doi: 10.1681/ASN.2013030314 
33. Pecoits-Filho R, Carvalho MJ, Stenvinkel $P$, Lindholm B, Heimburger O. Systemic and intraperitoneal interleukin-6 system during the first year of peritoneal dialysis. Perit Dial Int. (2006) 26:53-63. doi: 10.1177/089686080602600109

34. Liu SH, Li YJ, Wu HH, Lee CC, Lin CY, Weng CH, et al. High-sensitivity Creactive protein predicts mortality and technique failure in peritoneal dialysis patients. PloS ONE. (2014) 9:e93063. doi: 10.1371/journal.pone.0093063

35. Ikeda-Matsuo Y. The role of mPGES-1 in inflammatory brain diseases. Biol Pharm Bull. (2017) 40:557-63. doi: 10.1248/bpb.b16-01026

36. Pierre C, Guillebaud F, Airault C, Baril N, Barbouche R, Save E, et al. Invalidation of microsomal prostaglandin E synthase-1 (mPGES-1) reduces diet-induced low-grade inflammation and adiposity. Front Physiol. (2018) 9:1358. doi: 10.3389/fphys.2018.01358

37. Liu Y, Duan C, Chen H, Wang C, Liu X, Qiu M, et al. Inhibition of COX-2/mPGES-1 and 5-LOX in macrophages by leonurine ameliorates monosodium urate crystal-induced inflammation. Toxicol Appl Pharmacol. (2018) 351:1-11. doi: 10.1016/j.taap.2018.05.010

38. Wang Y, Tao J, Yao Y. Prostaglandin E2 activates NLRP3 inflammasome in endothelial cells to promote diabetic retinopathy. Horm Metab Res. (2018) 50:704-10. doi: 10.1055/a-0664-0699
39. Wu J, Li X, Zhu G, Zhang Y, He M, Zhang J. The role of resveratrol-induced mitophagy/autophagy in peritoneal mesothelial cells inflammatory injury via NLRP3 inflammasome activation triggered by mitochondrial ROS. Exp Cell Res. (2016) 341:42-53. doi: 10.1016/j.yexcr.2016.01.014

40. Zhang LL, Huang S, Ma XX, Zhang WY, Wang D, Jin SY, et al. Angiotensin (1-7) attenuated angiotensin II-induced hepatocyte EMT by inhibiting NOXderived H2O2-activated NLRP3 inflammasome/IL-1beta/Smad circuit. Free Radic Biol Med. (2016) 97:531-43. doi: 10.1016/j.freeradbiomed.2016.07.014

Conflict of Interest: The authors declare that the research was conducted in the absence of any commercial or financial relationships that could be construed as a potential conflict of interest.

Copyright (c) 2021 Luo, Hu, Zheng, Gong, Su, Ren, Ju, Jia and Dou. This is an open-access article distributed under the terms of the Creative Commons Attribution License (CC BY). The use, distribution or reproduction in other forums is permitted, provided the original author(s) and the copyright owner(s) are credited and that the original publication in this journal is cited, in accordance with accepted academic practice. No use, distribution or reproduction is permitted which does not comply with these terms. 